\title{
Oxidation of hexafluoropropylene to hexafluoropropylene oxide using oxygen
}

\author{
Monika Lągiewczyk, Zbigniew Czech* \\ "West Pomeranian University of Technology Szczecin, Institute of Organic Chemical Technology, ul. Pulaskiego 10, \\ 70-322 Szczecin, Poland, e-mail: psa_czech@wp.pl
}

\begin{abstract}
A method for pressure oxidation of hexafluoropropylene (HFP) to hexafluoropropylene oxide (HFPO), using oxygen, is presented. Oxidation was achieved in a batch-fed reactor at temperature range between 130 to $170^{\circ} \mathrm{C}$. The influence of temperature and kind of solvents, such as 1,2,2-trichloro-1,1,2-trifluoroethane (CFC-113) and carbon tetrachloride on the yield of HFPO and the course of oxidation was investigated in preliminary studies. The maximum HFPO yield of approximately $83 \%$ was noticed.
\end{abstract}

Keywords: hexafluoropropylene, hexafluoropropylene oxide, epoxidation.

\section{INTRODUCTION}

Hexafluoropropylene oxide (HFPO) is a valuable monomer that can be oligomerized to prepare intermediates for the synthesis of highly effective nonionic, ionic, and amphoteric surface-active agents. Even small amounts of such compounds cause a significant decrease in the surface tension of water, and, for this reason, these materials are used as components of extinguishing agents $\mathbf{1}$. HFPO oligomers are also employed as inert solvents and hydraulic fluids ${ }^{2}$. Higher oligomers are valuable as lubricating oils, showing good thermostability and chemical resistance ${ }^{3,4}$.

To date, HFPO has been produced by oxidation using sodium hypochlorite ${ }^{5}$, oxygen under pressure $^{6}$ and in a pressure-free method ${ }^{4}$, hydrogen peroxide ${ }^{7}$, and employing an electrochemical procedure ${ }^{1}$. HFPO can be obtained with high efficiency by epoxidation of HFP with hypochlorite using a phase-transfer catalyst in a system of two phases, water and an organic solvent, in the presence of an inorganic base. Quaternary ammonium, phosphonium, and arsenium salts, and lipophilic reagents complexed with sodium cations, have been used as phasetransfer catalysts. However, such methods are employed only in small-scale preparations $\mathbf{5}^{\mathbf{5}}$.

The oxidation of HFP by oxygen in the gaseous phase, in the presence of catalysts such as barium compounds, activated silica gels, metals (Ag, V), supported silica gel, or diatomaceous earth, has also been investigated ${ }^{4}$. Pressure oxidation of HFP using oxygen in the liquid phase shows the greatest promise for commercial production of $\mathrm{HFPO}^{8}$. Investigation of HFPO preparation by catalystfree oxidation using hydrogen peroxide in a water-soluble polar solvent such as acetone or acetonitrile is ongoing?.

Electrochemical oxidation has also attracted research interest1. An aqueous solution of acetonitrile, or a mixture thereof with acetic acid and a compound improving conductivity $\left(\mathrm{NaClO}_{4}\right)$, has been used as an electrolyte. The principal oxidation reaction of HFP, using oxygen, proceeds in both the gaseous and liquid phases as shown in Figure 1.

The reaction mechanism assumes that, after addition of oxygen to the double bond, a biradical is formed involving electrons of both oxygen and carbon ${ }^{5}$. The decomposition and rearrangement of this intermediate, together with decomposition of HFPO, results in the formation of sev-

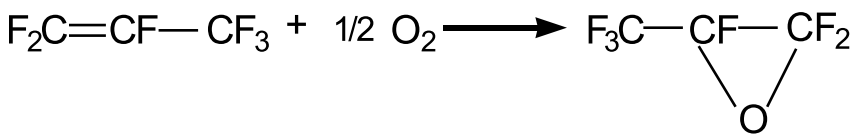

Figure 1. The principal reaction in oxidation of hexafluoropropylene

eral by-products including trifluoroacetic acid fluoride, carbonyl fluoride, 1-trifluoromethylperfluorocyclopropane, tetrafluoroethylene, and perfluorocyclopropane. Carbine is also formed temporarily. Figure 2 shows the relevant chemical equations ${ }^{\mathbf{1 0}}$.

The purpose of the present work was to investigate the influence of changes in technological parameters on direct oxidation of HFP using oxygen in a liquid phase under pressure, and to establish optimal parameters for process operation.
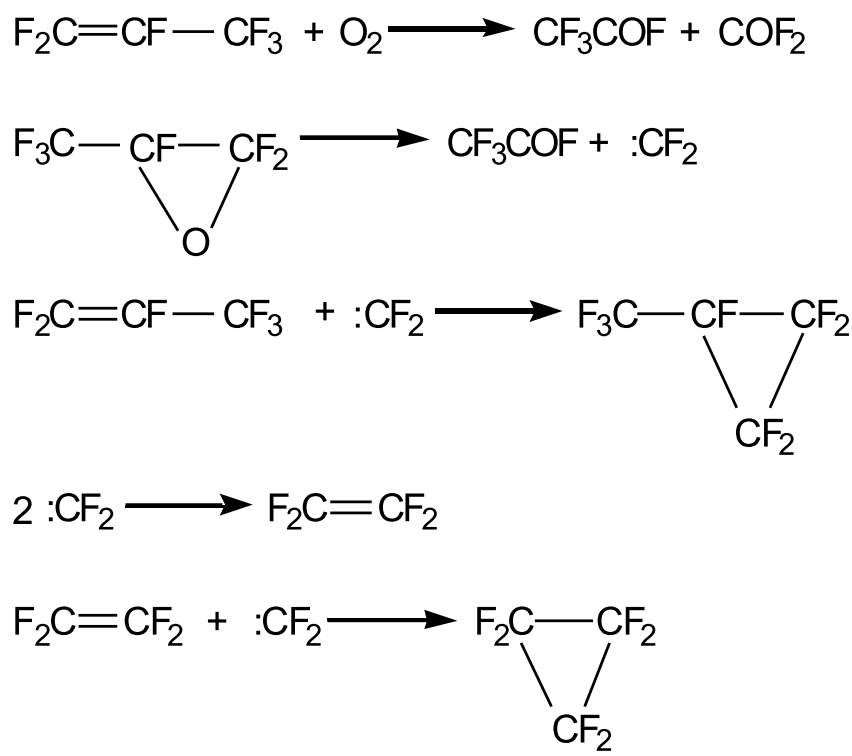

Figure 2. The mechanism of formation of HFPO

\section{EXPERIMENTAL}

\section{Raw Materials}

HFP was prepared by debromination of 1,2dibromohexafluoropropane. Oxygen and nitrogen were obtained from Messer-Poland. Carbon tetrachloride $\left(\mathrm{CCl}_{4}\right)$, perfluorotributylamine $\left(\left[\mathrm{CF}_{3}\left(\mathrm{CF}_{2}\right)_{3}\right]_{3} \mathrm{~N}\right)$, and 1,2,2- 
trichloro-1,1,2-trifluoroethane (CFC-113), were obtained from POCh, Poland.

\section{Oxidation Procedure and Analytical Methods}

The main instrumentation for oxidation of HFP is a reactor (volume $1.310 \mathrm{~cm}^{3}$ ) featuring a condenser in the upper level. The reactor was electrically heated, with manual regulation, and the feed voltage was controlled by an autotransformator. The reactor was equipped with temperature detectors and a manometer was installed at the top. The apparatus permitted addition of precise levels of olefins and oxygen, and featured an absorption system and a cryostat containing a cold trap, to obtain reaction products.

An appropriate amount of aprotic solvent was introduced to leak-proof the reactor. The reactor was operated in the temperature range $130 \ldots 170^{\circ} \mathrm{C}$, and an inert gas (nitrogen) was introduced to generate overpressure. Gaseous substrates (HFP and oxygen) were introduced at specified velocities. HFP was added to the reactor when the temperature was $70 \ldots 90^{\circ} \mathrm{C}$. Perfluoroolefin was added at a level greater than that of oxygen. Samples for analysis were obtained from three different sites during the chemical process. Products were collected from the reactor outlet (thus before absorption), after absorption but before outdropping, and in the outlet of the cold trap. Cryostat temperature was $-45^{\circ} \mathrm{C}$ and total reaction time was $1 \ldots 3 \mathrm{~h}$.

Fluoride ion levels in the eluate were determined potentiometrically after absorption of the gaseous products of oxidation. An Orion 4 STAR apparatus fitted with a fluoride electrode (model Orion 9609 B) was used for measurement.

Quantitative analysis of HFPO and the gaseous products of HFP oxidation was performed chromatographically using a Chrom 5 apparatus equipped with a thermal conductivity detector. The concentrations of gaseous HFP, HFPO, and trifluoroacetic acid fluoride were determined. A stainless steel column $(2.5 \mathrm{~m} \times 0.4 \mathrm{~cm})$ packed with silica gel ASTM-E of 80/100 mesh was used. Other detection conditions were as follows: carrier gas $\mathrm{H}_{2}=20$ $\mathrm{cm}^{3} / \mathrm{min}$, oven temperature $=150^{\circ} \mathrm{C}$ and injection level $=$ $100 \mu \mathrm{L}$.

\section{RESULTS AND DISCUSSION}

The influence of temperature on HFPO yield, in the range $130 \ldots 170^{\circ} \mathrm{C}$ (i.e. in the reaction zone), was explored under the following conditions: HFP $115 \mathrm{~g}(0.766 \mathrm{~mol})$, oxygen $9 \mathrm{dm}^{3}(0.4 \mathrm{~mol})$, carbon tetrachloride $2,042 \mathrm{~g}$ $\left(1,310 \mathrm{~cm}^{3}\right)$, and reaction time $3 \mathrm{~h}$. Oxygen was added in three aliquots (each of $3 \mathrm{dm}^{3}$ ) at $60 \mathrm{~min}$ intervals. The results are listed in Table 1.

It was found that, above $170^{\circ} \mathrm{C}$, the epoxy oxides formed were unstable and decomposed into by-products. At temperatures below ca. $155^{\circ} \mathrm{C}$, epoxidation of HFP to HFPO was not seen.

Table 2. The influence of solvent type on HFPO yield
Table 1. The influence of temperature on HFPO yield

\begin{tabular}{|c|c|c|c|c|c|}
\hline \multirow{2}{*}{$\begin{array}{c}\text { Reaction } \\
\text { time } \\
{[\mathrm{min}]}\end{array}$} & \multicolumn{2}{|c|}{ Reactor temperature $\left[{ }^{\circ} \mathrm{C}\right]$} & pressure & yield \\
\cline { 2 - 5 } & top & middle & bottom & & {$[\%]$} \\
\hline 20 & 126 & 136 & 145 & 34.5 & - \\
40 & 145 & 151 & 157 & 35.5 & - \\
60 & 152 & 157 & 162 & 36.0 & - \\
80 & 158 & 162 & 166 & 37.0 & 8.5 \\
100 & 160 & 164 & 167 & 36.0 & 21.3 \\
120 & 160 & 164 & 169 & 36.2 & 30.1 \\
140 & 161 & 166 & 170 & 36.4 & 41.0 \\
160 & 158 & 164 & 170 & 36.6 & 48.6 \\
180 & 170 & 172 & 174 & 36.8 & 43.9 \\
\hline
\end{tabular}

The influence of three solvents on the oxidation of HFP to HFPO was also investigated. The experimental parameters were a reaction time of $3 \mathrm{~h}$, a temperature of $160 \ldots 170^{\circ} \mathrm{C}$, a molar ratio of $\mathrm{HFP} / \mathrm{O}_{2}$ of $1: 1.05$, and three oxygen aliquots each of $3 \mathrm{dm}^{3}$. The data obtained are listed in Table 2.

The best results were obtained with use of the solvent 1,2,2-trichloro-1,1,2-trifluoroethane (CFC-113), with an HFPO yield of approximately $83 \%$. The lowest yield (44\%) was obtained when carbon tetrachloride was employed. This is attributable to the low solubility of both oxide and HFP in this solvent.

\section{CONCLUSIONS}

Temperature plays the most significant role in oxidation of HFP to HFPO. To obtain HFPO in good yield it is necessary to conduct the oxidation reaction in the temperature range $160 \ldots 170^{\circ} \mathrm{C}$. Yield can be improved by the use of an appropriate aprotic solvent. The best solvent for oxidation of HFP was 1,2,2-trichloro-1,1,2-trifluoroethane (CFC-113), which afforded the product in $83 \%$ yield.

Synthesis of HFPO is both complicated and time-consuming. The oxidation reaction can be very violent if the oxygen feed level is too fast. The selection of appropriate process parameters including temperature and molar ratio of $\mathrm{HFP} / \mathrm{O}_{2}$ render the temperature controllable and limit formation of by-products.

\section{LITERATURE CITED}

1. Fittes, D.W., Griffiths, D.J. \& Nash, P. (1969). The use of light water for major aircraft fires. Fine Technology 5 (4), 284 - 298. DOI: 10.1007/BF02600416.

2. Millauer, H. (1977). Process for the preparation of hexafluoropropylene epoxide. US Pat. 4014762.

3. Hill, J.T., Garabedian, M.E. (1972). Process for preparing fluorine-containing polymers. US Pat. 3660315.

4. Atkins, G. (1973). Process for the epoxidation of hexafluoropropylene. US Pat. 3775439.

5. Ikeda, M., Miura, M. \& Aoshima, A. (1990). Process for the production of hexafluoropropylene oxide. US Pat. 4902810.

6. Meissner, E. \& Wróblewska, A. (2007). Oxidation of hexafluoropropylene with molecular oxygen. Polish J. Chem. Technol. 9 (1), 20 - 22. DOI: 10.2478/v10026-007-0006-y.

\begin{tabular}{|l|c|c|c|c|c|c|c|}
\hline \multirow{2}{*}{ Type of solvent } & solvent & \multicolumn{2}{|c|}{ HFP } & \multicolumn{2}{|c|}{$\mathrm{O}_{2}$} & \multicolumn{2}{c|}{ Temperature } \\
\cline { 2 - 6 }$[? \mathrm{C}]$ & $\begin{array}{c}\text { Yield } \\
{[\%]}\end{array}$ \\
\hline $\mathrm{CCl}_{4}$ & {$\left[\mathrm{~cm}^{3}\right]$} & {$[\mathrm{g}]$} & {$[\mathrm{moles}]$} & {$\left[\mathrm{dm}^{3}\right]$} & {$[\mathrm{moles}]$} & 163 & 44 \\
{$\left[\mathrm{CF}_{3}\left(\mathrm{CF}_{2}\right)_{3}\right]_{3} \mathrm{~N}$} & 1.310 & 115 & 0.766 & 9 & 0.4 & 165 & 59 \\
$\mathrm{CFC}-113$ & 1.310 & 115 & 0.766 & 9 & 0.4 & 167 & 83 \\
\hline
\end{tabular}


7. Meissner, E. \& Wróblewska, A. (2006). Oxidation of hexafluoropropylene using hydrogen peroxide in the presence of phase transfer catalyst. Polish J. Chem. Technol. $8(2), 66-67$.

8. Carlos, D.P. (1970). Method for the preparation of halogenated epoxides. US Pat. 3536733.

9. Eleuterio, H.S. \& Meschke, R.W. (1967). Fluorocarbon epoxides. US Pat. 3358003.

10. Gilbert, J.R., Slagle, I.R., Graham, R.E. \& Gutman, D. (1976). Direct identification of reactive routes and measurement of rate constants in the reactions of oxygen atoms with the fluoroethylenes. J. Phys. Chem. 80 (1), 14 - 18. DOI: $10.1021 / \mathrm{j} 100542 \mathrm{a} 003$. 